\title{
Nuovo approccio nel trattamento dell'ipertensione arteriosa resistente mediante anastomosi artero-venosa
}

\author{
Federico Pieruzzi ${ }^{1}$, Federica Mescia² \\ ${ }^{1}$ Università degli Studi di Milano Bicocca, Dipartimento di Scienze della Salute, Clinica Nefrologica, AO San Gerardo di Monza, Monza (MB) \\ ${ }^{2}$ Università degli Studi di Milano Bicocca, Scuola di Specializzazione in Nefrologia, Ospedale Papa Giovanni XXIII, Bergamo
}

\begin{abstract}
A novel approach for the treatment of resistant hypertension
Interventional approaches to lower blood pressure such as baroreflex activation therapy and renal sympathetic denervation have been developed and tested clinically. These two approaches are based on sound pathophysiological concepts and target mechanisms highly relevant to the control of blood pressure and cardiovascular disease, although recent evidences shed some shadows on their effectiveness and large scale use in clinical practice. New interventional approaches based on different mechanisms are emerging. The ROX CONTROL HTN study is an open-label, multicenter, prospective randomised trial assessing the effects of creating a central arteriovenous anastomosis of $4 \mathrm{~mm}$ diameter between the distal iliac vein and artery with an arteriovenous coupler device. This arteriovenous anastomosis was associated with a significant and sustained reduction of both diastolic and systolic blood pressure values. Technical success of the procedure was assessed by an immediate drop in blood pressure after the creation of the anastomosis. The reduction of peripheral resistance could be the mechanisms of the blood lowering effect, although it was not directly tested in the study. In addition to the concern about the safety of this new interventional procedure, due to the elevated number of local side effects, the absence of adverse consequences of central arteriovenous anastomosis on cardiac output and central haemodynamics also need to be shown.
\end{abstract}

This approach might be a useful adjunctive therapy for patients with uncontrolled hypertension.

Keywords: Central arteriovenous anastomosis, Resistant hypertension, Coupler device, Blood pressure

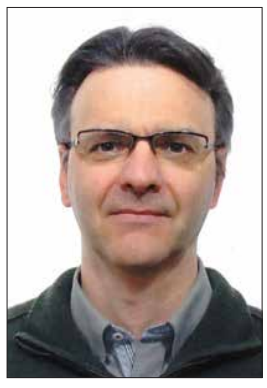

La ricerca di approcci terapeutici innovativi per l’ipertensione arteriosa refrattaria è un settore più vivace che mai, nonostante i risultati deludenti del più recente grande trial sulla denervazione renale, lo studio SYMPLICITY HTN-3, che, confrontando il trattamento con una procedura "sham", non ha confermato i risultati positivi dei due studi precedenti (1). Infatti una nuova procedura interventistica per la terapia Federico Pieruzzi dell'ipertensione arteriosa refrattaria è stata proposta recentemente. Si tratta del confezionamento di un'anastomosi

Accepted: April 2, 2015

Published online: May 11, 2015

Indirizzo per la corrispondenza:

Dr. Federico Pieruzzi

Dipartimento di Scienze della Salute

Università degli Studi di Milano Bicocca

Clinica Nefrologica, AO San Gerardo di Monza

Via Pergolesi 33

20900 Monza (MB)

federico.pieruzzi@unimib.it artero-venosa centrale grazie a un device, il "ROX Coupler", ideato dalla start-up biomedica ROX Medical (California, USA). Attingendo da tecnologie e metodiche maturate dalla radiologia e dalla cardiologia interventistica, è stata messa a punto una procedura percutanea che permette la creazione di un'anastomosi artero-venosa del diametro di 4 millimetri tra arteria e vena iliaca esterna distali mediante il posizionamento del "ROX Coupler", una sorta di stent endovascolare. La manovra dura meno di un'ora, viene effettuata in anestesia locale, sotto guida fluoroscopica, e richiede il posizionamento di due introduttori, uno in arteria a l'altro in vena femorale (per ulteriori dettagli tecnici, è possibile visionare l'animazione creata dai produttori al sito http://www.roxmedical.com/about-rox/flow-procedure-video) (Fig. 1).

L'applicazione di questo device nel campo dell'ipertensione refrattaria deriva da una precedente esperienza: la procedura è stata inizialmente ideata per alleviare la dispnea in pazienti con grave broncopneumopatia cronica ostruttiva. Durante queste prime esperienze cliniche, il ragguardevole, e non ricercato, effetto antipertensivo è stato presto incidentalmente documentato e, quindi, studiato ad hoc, fino al successivo sviluppo del "ROX Coupler". II meccanismo d'azione della procedura, alla base dell'effetto antipertensivo, consiste verosimilmente nel ridurre le resistenze arteriose periferiche 


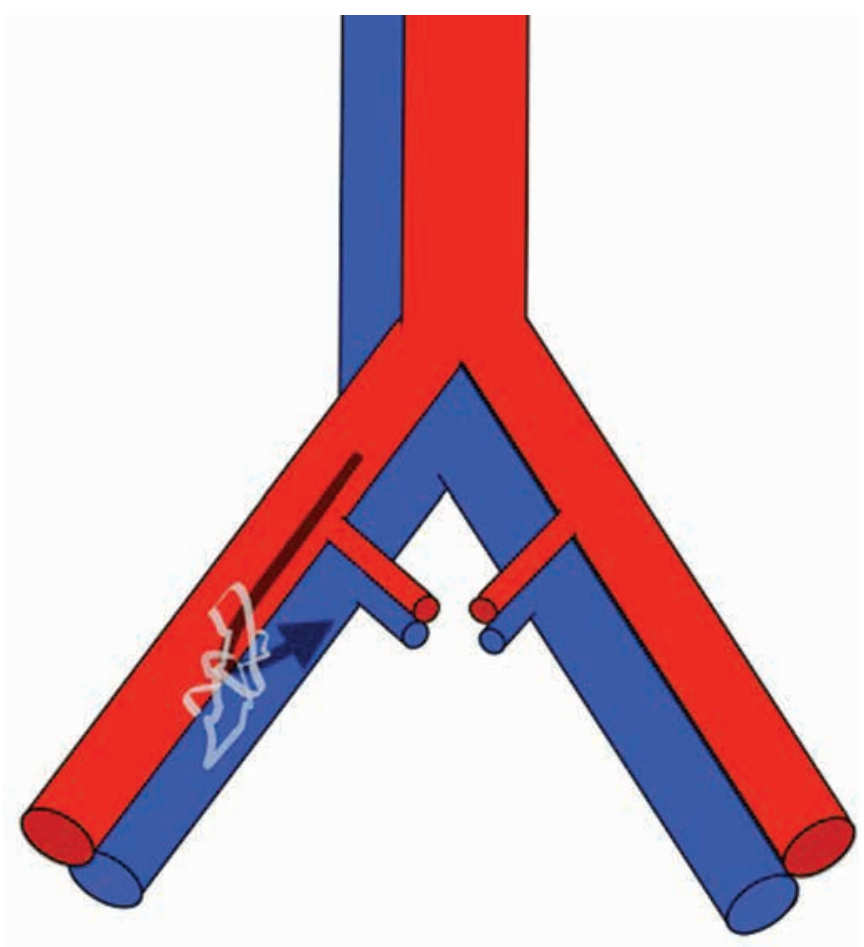

Fig 1. - Illustrazione schematica del posizionamento del ROX Coupler.

mediante l'aggiunta all'albero arterioso di un segmento venoso a bassa resistenza e a elevata compliance, in modo da sfruttarne gli effetti meccanici sul circolo.

Melvin Lobo et al. hanno recentemente pubblicato sul Lancet i primi risultati relativi all'uso di questo device nella pratica clinica, nell'ambito del ROX CONTROL HTN study (2). Si tratta di uno studio prospettico, multicentrico, randomizzato e open-label, sponsorizzato dalla ROX Medical. Nel periodo tra Ottobre 2012 e Aprile 2014 sono stati reclutati e randomizzati 83 pazienti di età compresa tra i 18 e gli 80 anni in 16 centri europei. II criterio di inclusione fondamentale era l'ipertensione refrattaria, definita come pressione sistolica al di sopra di $140 \mathrm{mmHg}$ in ambulatorio e pressione media superiore a $135 / 85 \mathrm{mmHg}$ al monitoraggio Holter nelle 24 ore, nonostante l'assunzione di almeno tre farmaci antipertensivi di diverse classi, incluso un diuretico. Sono stati esclusi pazienti affetti da insufficienza renale di grado moderato-avanzato (eGFR stimato secondo MDRD $<30 \mathrm{~mL} / \mathrm{min} / 1.73 \mathrm{~m}^{2}$ ), con diabete mellito tipo 1 , storia di recente cardiopatia ischemica o vasculopatia cerebrale, grave malattia vascolare periferica arteriosa o venosa e ipertensione polmonare o sottoposti a denervazione renale nei 6 mesi precedenti.

I pazienti sono stati randomizzati a essere sottoposti alla procedura e a continuare ad assumere l'abituale terapia medica (casi) oppure a proseguire con il solo trattamento farmacologico (controlli). L'analisi conclusiva ha incluso 42 casi e 35 controlli. A 6 mesi di follow-up, i pazienti sottoposti alla procedura hanno mostrato una riduzione media della pressione arteriosa sistolica di $26.9 \mathrm{mmHg}(p<0.0001)$ in ambulatorio e di $13.5 \mathrm{mmHg}(\mathrm{p}<0.0001)$ al monitoraggio nelle 24 ore, a fronte di piccole variazioni non statisticamente significative nei pazienti del gruppo di controllo. Un andamento analogo è stato rilevato per la pressione diastolica a 6 mesi dalla procedura (in ambulatorio riduzione media della pressione diastolica nei casi di $20.1 \mathrm{mmHg}, \mathrm{p}<0.0001$, vs. 2.4 $\mathrm{mmHg}, \mathrm{p}=0.26$ nei controlli; al monitoraggio nelle 24 ore riduzione media di $13.5 \mathrm{mmHg}, \mathrm{p}<0.0001$, nei casi vs. $0.1 \mathrm{mmHg}$, $p=0.96$, nei controlli). Gli Autori riportano un'efficacia immediata della procedura, con un'istantanea significativa riduzione dei valori pressori appena dopo la creazione dell'anastomosi arterovenosa. I risultati sono sovrapponibili anche nella sottoanalisi effettuata sui 20 pazienti, equamente distribuiti nei due gruppi, che erano stati precedentemente sottoposti, da almeno 6 mesi, a procedure di denervazione renale senza alcun beneficio.

Inoltre, durante il follow-up, 11 pazienti sottoposti alla procedura e solo 2 pazienti nel gruppo di controllo hanno ridotto la terapia antipertensiva $(p=0.03)$ e sono state registrate 5 ospedalizzazioni per crisi ipertensive, tutte in 3 pazienti del gruppo di controllo.

Gli Autori hanno segnalato 13 eventi avversi correlabili alla procedura endovascolare, come dissecazione arteriosa, reazione al mezzo di contrasto, anemizzazione o trombosi venosa profonda. È stata riportata anche un'elevata incidenza (12 casi, 29\% dei pazienti) di stenosi venosa iliaca in sede prossimale all'anastomosi. I pazienti affetti erano sintomatici per edema dell'arto omolaterale e, in alcuni casi, aumento dei valori di pressione arteriosa; la diagnosi è stata fatta tra 2.3 e 8.7 mesi dopo la procedura e in tutti i casi sono stati effettuati una venoplastica percutanea e un eventuale posizionamento di stent venoso, con successo.

Ci troviamo di fronte alla nuova frontiera del trattamento dell'ipertensione arteriosa refrattaria o all'ennesimo promettente device ma ancora privo di una solidità clinica e scientifica?

\section{Efficacia}

Come riconosciuto dagli Autori stessi, si tratta di un lavoro preliminare, con diverse limitazioni che non permettono di trarre conclusioni definitive in merito all'efficacia del trattamento e di proporne un'immediata applicazione nella pratica clinica. In primis, nonostante i risultati ottenuti siano statisticamente fortemente significativi, il numero di pazienti reclutati è molto limitato e manca un gruppo di controllo ideale, sottoposto a procedura "sham". Inoltre, la terapia farmacologica assunta dai pazienti dello studio non è stata uniformata e l'aderenza al trattamento medico non è stata verificata in alcun modo.

\section{Sicurezza}

Complessivamente, più della metà dei pazienti sottoposti alla procedura ha riportato un evento avverso. Nel $31 \%$ dei casi si è trattato di problematiche insorte durante la manovra o nel periodo immediatamente successivo e 
intrinsecamente legate alle metodiche di accesso endovascolare. Da notare che la necessità di ottenere contestualmente un doppio accesso vascolare, arterioso e venoso, moltiplica inevitabilmente la probabilità di andare incontro a complicanze procedurali. È probabile che in futuro, con l'applicazione della procedura su una più ampia casistica e con l'acquisizione di maggiore esperienza da parte degli operatori, l'incidenza di tali rischi si modifichi. Tuttavia, le complicanze tecniche saranno difficilmente azzerabili e costituiranno sempre un importante fattore da considerare nel bilancio costi-benefici da effettuare prima di porre indicazione alla procedura.

A nostro avviso ancora più meritevole di attenzione è il dato relativo all'elevata incidenza, del $29 \%$, di stenosi venose a insorgenza tardiva nei pazienti sottoposti alla procedura. Tale complicanza, peculiare della procedura di anastomosi artero-venosa, ha richiesto in tutti i casi di sottoporre i pazienti a un'ulteriore manovra invasiva e, con una frequenza non specificata nel lavoro, ha limitato l'efficacia antipertensiva del trattamento. I dati attualmente disponibili sono estremamente limitati e sono chiaramente indispensabili informazioni su una casistica più ampia e con un follow-up più prolungato. Nell'ambito del breve periodo di osservazione dello studio, gli Autori riportano un caso di stenosi fino a 8.7 mesi dalla procedura. Per quanto tempo effettivamente sussiste un aumentato rischio di stenosi venosa post-manovra? È possibile andare incontro a restenosi post-venoplastica e/o stenting? Tali rischi sono passibili di modulazione, per esempio con terapie antitrombotiche in profilassi primaria e/o secondaria?

Il lavoro non include poi alcuna valutazione formale degli effetti emodinamici secondari alla creazione dell'anastomosi artero-venosa centrale, altro possibile motivo di importanti eventi avversi. Attingendo alla letteratura relativa all'impatto cardiovascolare del confezionamento di fistole artero-venose per emodialisi, gli Autori ipotizzano che il rischio di scompenso cardiaco legato alla procedura sia molto contenuto e che anzi la riduzione delle resistenze periferiche indotta dalla manovra possa essere emodinamicamente favorevole. II "ROX Coupler" creerebbe uno shunt con flussi massimi di $0.8-1.2 \mathrm{~mL} / \mathrm{min}$, ben al di sotto quindi di $2 \mathrm{~L} / \mathrm{min}$, valore che è stato identificato come soglia critica per indurre un rischio consistente di scompenso cardiaco a elevata portata nei portatori di fistola artero-venosa (3). Sono chiaramente necessari dati emodinamici e clinici di follow-up per avvalorare o meno questa ipotesi.

Come notato anche da Markus Schlaich e Dagmara Hering nell'editoriale di accompagnamento allo studio (4), è stata inoltre osservata una marcata riduzione della pressione diastolica post-procedura, di entità quasi sovrapponibile all'abbassamento della pressione sistolica. Tale risposta, probabilmente indissolubilmente legata alla modulazione delle resistenze periferiche indotta dal trattamento, potrebbe essere potenzialmente svantaggiosa soprattutto in pazienti affetti da cardiopatia ischemica e con ipertensione sistolica isolata. Questi aspetti andranno accuratamente valutati nell'estensione del follow-up e in ulteriori studi clinici.

\section{One size fits all?}

Infine, non possiamo dimenticare che il meccanismo d'azione con cui il "ROX Coupler" induce una riduzione dei valori pressori è stato solo ipotizzato, ma non provato. È assolutamente verosimile che il confezionamento di uno shunt artero-venoso aumenti artificiosamente la compliance arteriosa, con ripristino dell' "effetto Windkessel", riduzione delle resistenze periferiche e conseguente effetto antipertensivo. Tuttavia, questa ipotesi non è stata formalmente verificata, né sono stati effettuati studi meccanicistici di alcun tipo che esplorino altri possibili effetti anti-ipertensivi indotti dalla procedura. L'esperienza su eventuali effetti ipotensivi secondari a creazioni di anastomosi artero-venose è limitata. Le fistole arterovenose (FAV) degli arti superiori utilizzate per la dialisi in genere non determinano significative e diffuse alterazioni dei valori di pressione arteriosa sistemica sisto-diastolica, sebbene in alcuni pazienti siano state segnalate la normalizzazione dei valori pressori o la necessità di sospendere la terapia antipertensiva dopo la creazione della FAV. È probabile che la marcata differenza della portata dell'arteria femorale (8 $\mathrm{L} / \mathrm{min})$ rispetto alla brachiale $(80-120 \mathrm{~mL} / \mathrm{min})$ o alla radiale $(50$ $\mathrm{mL} / \mathrm{min}$ ), associata alla differente estensione del letto vascolare dei due distretti, abbia un ruolo importante nel determinare un significativo calo delle resistenze periferiche nel caso del distretto femorale rispetto a quello brachiale/radiale.

Sarà cruciale raccogliere maggiori informazioni su tali aspetti, non solo per arricchire le nostre conoscenze relative alla fisiopatologia dell'ipertensione, ma anche per valutare meglio i potenziali effetti negativi legati a questa procedura e per identificare possibili fattori predittivi positivi o negativi di risposta. Come ci sta insegnando anche l'esperienza della denervazione renale, il successo terapeutico nei casi complessi di ipertensione refrattaria non può che derivare da un approccio multitarget e supportato da una solida base fisiopatologica, ma anche confezionato su misura per il singolo paziente.

\section{Disclosures}

Financial support: No financial support was received for this submission. Conflict of interest: None of the authors has conflict of interest with this submission.

\section{Bibliografia}

1. Bhatt DL, Kandzari DE, O'Neill WW, et al. A Controlled Trial of Renal Denervation for Resistant Hypertension. N Engl J Med 2014;370(15):1393-401.

2. Lobo MD, Sobotka PA, Stanton A, et al. Central arteriovenous anastomosis for the treatment of patients with uncontrolled hypertension (the ROX CONTROL HTN study): a randomised controlled trial. Lancet. 2015 Apr 25;385(9978):1634-41.

3. Basile C, Lomonte C, Vernaglione L, Casucci F, Antonelli M, Losurdo $\mathrm{N}$. The relationship between the flow of arteriovenous fistula and cardiac output in haemodialysis patients. Nephrol Dial Transplant 2008;23(1):282-7.

4. Schlaich M, Hering D. Central arteriovenous anastomosis in resistant hypertension? Lancet. 2015 Apr 25;385(9978):1596-7. 